Logic and Logical Philosophy

Volume 3 (1995), 87-100

Janusz Czelakowski

\title{
LOGICS AND OPERATORS
}

CONTENTS

1. Introduction

2. A hierarchy of logics based

on the Leibniz operator

3. Protoalgebraic logics

4. Equivalential logics

5. Final remarks

Received October 25, 1995 


\section{Introduction}

A logic (= deductive system) is any pair $\langle S, C\rangle$, where $S$ is a sentential language (i. e., a countable absolutely free algebra freely generated by a counably infinite set of free generators $V$ (sentential variables) and endowed with a countable set of finitary operations (logical connectives)) and $C$ is a structural consequence operation on $S$.

Two connectives are of special interest in metalogical investigations the connective of implication which is important due to its connections to the notion of inference, and the connective of equivalence. The latter connective expresses, in the material sense, the fact that two sentences have the same logical value while in the strict sense it expresses the fact that two sentences are interderivable on the basis of a given logic. The process of identification of equivalent sentences relative to theories of a logic $C$ defines a class of abstract algebras. The members of the class are called Lindenbaum-Tarski algebras of the $\operatorname{logic} C$. One may abstract from the origin of these algebras and examine them by means of purely algebraic methods.

This approach to the methodology of the deductive systems, based on Lindenbaum-Tarski algebras, is particularly important because it bridges the gap between logic and algebra and makes it possible to apply the powerful methods of contemporary algebra in metalogic. This approach has initiated the discussion on the scope of algebraic methods in metalogical investigations and has led to interesting generalizations of the Lindenbaum-Tarski method. One of the results of this discussion is a clarification of the concept of an algebraizable logic (Blok and Pigozzi [1989]). Roughly speaking, a logic is algebraizable if, in a natural way, a certain class of algebras can be associated with this logic and, moreover, the properties of this logic are fully reducible to the purely algebraic properties of the associated class of algebras.

The Lindenbaum-Tarski method enables one to algebraize a number of logics as e.g. classical or intuitionistic system, and many-valued logics. For example, if $C$ is the classical consequence (logic), then it is well-known that the relation:

$$
\alpha \equiv_{T} \beta \Longleftrightarrow \alpha \leftrightarrow \beta \in C(T)
$$

defines, for any theory $T \subseteq S$, a congruence of the algebra of sentences $S$. The resulting class of Lindenbaum-Tarski algebras, obtained by factoring the algebras of sentences by the congruence $(*)$, coincides with the class of 
Boolean algebras. In turn, the class of Lindenbaum-Tarski algebras corresponding to intuitionistic logic coincides with the class of Heyting algebras.

The question about the scope of the above method arises. There are numerous examples of logics to which the Tarski's method cannot be directly applied since there may not exist a connective $\leftrightarrow$ in the language $S$ of the logic which defines, according to formula $(*)$, a congruence on the language. Prucnal and Wroński [1974] have proposed a generalization of the Lindenbaum-Tarski technique by replacing the equivalence connective by a possibly infinite set of sentential formulas which collectively possesses many properties of the equivalence connective. Any logic which has such a set is called equivalential. In a more formal rendering, a logic $C$ is equivalential (finitely equivalential) if there exists a set (a finite set) $E(p, q)$ of sentential formulas in two variables $p$ and $q$ such that, for each theory $T$, the relation $\equiv_{T}$, where

$$
\alpha \equiv_{T} \beta \Longleftrightarrow E(p, q) \subseteq C(T),
$$

is a congruence on the language $S$ compatible with $T$. The notion of an equivalential logic turns out to be very useful in the analysis of intensional logics such as modal, temporal, or dynamic logics.

A wider perspective is offered by the approach based on the concept of the Leibniz operator $\boldsymbol{\Omega}$, the term introduced by Blok and Pigozzi [1986]. (The notion is in fact much older; it is implicit e.g. in the work of Wójcicki [1973], see also Łoś [1949].) The definition of the Leibniz operator is independent from one or any other logic admitted in the language $S$. The Leibniz operator $\Omega$ is a function which assigns to each theory $T \subseteq S$ a congruence on $S$, denoted by $\Omega T$. The congruence $\Omega T$ is the synonymy relation on $S$ relative to $T$. Thus

$$
\alpha \equiv \beta(\boldsymbol{\Omega} T) \Longleftrightarrow \bigwedge_{\varphi \in S} \bigwedge_{p \in \operatorname{Var}(\varphi)}(\varphi(p / \alpha) \in T \Leftrightarrow \varphi(p / \beta) \in T),
$$

where $\operatorname{Var}(\varphi)$ is the set of variables occurring in $\varphi$, and $\varphi(p / \alpha)$ is the result of simultaneously replacing the variable $p$ in $\varphi$ by the sentence $\alpha$.

$\boldsymbol{\Omega} T$ is the largest congruence on $S$ compatible with $T$, i.e., $\boldsymbol{\Omega} T$ is the largest of all congruences $\Phi$ on $S$ with the property that $\alpha \equiv \beta(\Phi)$ implies that $\alpha \in T$ iff $\beta \in T$, for every pair $\alpha, \beta$. The definition of $\boldsymbol{\Omega} T$ is related to the well-known method of defining the equality relation in second-order logic that goes back to Leibniz. For this reason $\boldsymbol{\Omega} T$ is called the Leibniz congruence associated with $T$, and the operator $\boldsymbol{\Omega}$, assigning the congruence $\boldsymbol{\Omega} T$ to each theory $T$ in $S$, is called the Leibniz operator (Blok and Pigozzi [1986]). The operator $\Omega$ is then extended in a natural way to the power set 
$\wp(A)$ of an arbitrary algebra $A$ similar to the language $S$, where it is denoted by $\boldsymbol{\Omega}_{A}$. More specifically, for every $D \subseteq A$ :

$$
\bigwedge_{\varphi\left(x, u_{1}, \ldots, u_{n}\right) \in S} \bigwedge_{\left\langle e_{1}, \ldots, e_{n}\right\rangle \in A^{n}}\left(\varphi\left(a, e_{1}, \ldots, e_{n}\right) \in D \Leftrightarrow \varphi\left(b, e_{1}, \ldots, e_{n}\right) \in D\right) .
$$

In the context of metalogic the format of the operator $\Omega$ is restricted by admitting that the domain of $\boldsymbol{\Omega}$ is the family of all theories of a given $\operatorname{logic} C$. The so restricted Leibniz operator thus assigns the congruence $\boldsymbol{\Omega} T$ to each closed theory $T \in T h(C)$. At the same time the domain of $\boldsymbol{\Omega}_{A}$ is narrowed, for each algebra $A$, to the closure system $F i_{C}(A)$ of all $C$-filters (alias deductive filters) on $A$.

\section{A hierarchy of logics based on the Leibniz operator}

In the general case, there are no regularities in the run of $\boldsymbol{\Omega}$ on $T h(C)$. The definition of $\boldsymbol{\Omega}$ suggests however the idea of distinguishing some plausible properties of the operator. These properties, though not universally valid, may be eventually satisfied for particular logics. This remark, in turn, opens the possibility of building a certain natural hierarchy of logics based on definite properties of the operator $\boldsymbol{\Omega}$. This is the core of the operator approach to the problem of typology of logical systems. Blok and Pigozzi [1986] must be credited with acknowledging the importance of the Leibniz operator and its properties in metalogical research. The monotonicity property of $\boldsymbol{\Omega}$ (on $T h(C)$ ) serves as a basis for distinguishing the class of protoalgebraic logics (see the remarks below). The continuity property of $\boldsymbol{\Omega}$ is equivalent to finite equivalentiality of $C$. These observations have initiated the operator approach as the basis for a typology of deductive systems. A fully fledged typology of protoalgebraic deductive systems was presented in Czelakowski's work [1992].

Generally, an operator on a language $S$ is any function $\boldsymbol{O}$ which assigns a congruence on $S$ to each theory on $S$. In the context of metalogic, the run of any operator is being always restricted to the closed theories of a given $\operatorname{logic} C$.

The hierarchy of deductive systems outlined below directly refers to the following list of (possible) properties of an arbitrary operator $\boldsymbol{O}$. Here $C$ is assumed to be a fixed sentential logic and $T, T_{1}, T_{2}, T_{i}(i \in I)$ range over arbitrary theories of $C$. 
monotonicity

injectivity

continuity

meet-continuity

commutativity with

inverse substitutions
$T_{1} \subseteq T_{2}$ implies $\boldsymbol{O} T_{1} \subseteq \boldsymbol{O} T_{2}$;

$\boldsymbol{O} T_{1}=\boldsymbol{O} T_{2}$ implies $T_{1}=T_{2}$;

$\boldsymbol{O} \bigcup\left\{T_{i}: i \in I\right\}=\bigcup\left\{\boldsymbol{O} T_{i}: i \in I\right\}$ for all directed system $T_{i}(i \in I)$ such that the union $\bigcup\left\{T_{i}: i \in I\right\}$ is a theory of $C$;

$\boldsymbol{O} \bigcap\left\{T_{i}: i \in I\right\}=\bigcap\left\{\boldsymbol{O} T_{i}: i \in I\right\}$;

$\boldsymbol{O} e^{-1} T=e^{-1} \boldsymbol{O} T$ for every substitution $e$.

\section{Protoalgebraic logics}

Definition 1. A logic $\langle S, C\rangle$ is protoalgebraic iff the operator $\Omega$ is monotonic on $T h(C)$.

We shall give a list of properties equivalent to protoalgebraicity. Suppose that among the connectives of the language $S$ there is a binary connective $\rightarrow$ (an arrow). Most of the familiar logics possess the following two properties:

$$
p \rightarrow p \in C(\emptyset)
$$

and

$$
q \in C(\{p, p \rightarrow q\})
$$

i.e., the law of identity $p \rightarrow p$ and the rule detachment $p, p \rightarrow q / q$ are valid in $C$. The two properties are generalized as follows. Let $P(p, q)$ be a set of sentences of $S$ in two variables $p$ and $q$. The conditions

$$
\begin{aligned}
& P(p, p) \subseteq C(\emptyset) \\
& q \in C(\{p\} \cup P(p, q))
\end{aligned}
$$

are called the reflexivity and Modus Ponens properties for the logic $C$ relative to $P$.

Let $E=E(p, q, \underline{r})$ be a possibly infinite set of sentences of $S$ built up from the variables $p, q$ and possibly other variables $\underline{r}=\left\langle r_{1}, r_{2}, \ldots\right\rangle$ called parameters. Let $k=|\underline{r}|$ be the length of the string $\underline{r}$. Thus $k \leqslant \omega$.

$E(p, q, \underline{r})$ is called a parameterized system of equivalence sentences for a logic $\langle S, C\rangle$ (a parameterized equivalence for $C$, for short) if the following three conditions are satisfied: 
$\mathrm{p}-(\mathrm{R}) \quad E(p, p, \underline{r}) \subseteq C(\emptyset)$;

$\mathrm{p}-(\mathrm{MP}) \quad q \in C\left(\{p\} \cup \bigcup\left\{E(p, q, \underline{\gamma}): \underline{\gamma} \in S^{k}\right\}\right)$;

$\mathrm{p}-\left(\mathrm{RM}_{\text {sim }}\right) \quad$ for each $n$-ary connective $f$ of $S(n \geqslant 0)$ :

$$
\begin{array}{r}
\bigcup\left\{E\left(f\left(p_{1}, \ldots, p_{n}\right), f\left(q_{1}, \ldots, q_{n}\right), \underline{\gamma}\right): \underline{\gamma} \in S^{k}\right\} \subseteq \\
C\left(\bigcup\left\{E\left(p_{i}, q_{i}, \underline{\gamma}\right):: i=1, \ldots, n \& \underline{\gamma} \in S^{k}\right\}\right) .
\end{array}
$$

It can be shown that the conditions $\mathrm{p}-(\mathrm{R}), \mathrm{p}-(\mathrm{MP})$ and $\mathrm{p}-\left(\mathrm{RP}_{\text {sim }}\right)$, the last one referred to as simple replacement, jointly imply the conditions:

$\mathrm{p}-(\mathrm{S}) \quad \bigcup\left\{E(p, q, \underline{\gamma}): \underline{\gamma} \in S^{k}\right\} \subseteq C\left(\bigcup\left\{E(q, p, \underline{\gamma}): \underline{\gamma} \in S^{k}\right\}\right)$

$\mathrm{p}-(\mathrm{T}) \quad \bigcup\left\{E(p, r, \underline{\gamma}): \underline{\gamma} \in S^{k}\right\} \subseteq$

$$
C\left(\bigcup\left\{E(p, \bar{q}, \underline{\gamma}) \stackrel{-}{\gamma} \in S^{k}\right\}\right) \cup C\left(\bigcup\left\{E(q, r, \underline{\gamma}): \underline{\gamma} \in S^{k}\right\}\right)
$$

of parameterized symmetry and parameterized transitivity.

If $M=\langle A, D\rangle$ is a matrix for $S$, and $C$ is a logic in $S$, then $F i_{C}(M)$ stands for the set of all $C$-filters in $M$, i.e.,

$$
F i_{C}(M):=\left\{F \in F i_{C}(A): D \subseteq F\right\}
$$

Suppose $h: M \rightarrow N$ is a strict homomorphism between models of $C$. Then obviously $F \subseteq h^{-1} h(F)$ for every $C$-filter $F \in F i_{C}(M)$.

Definition 2. A logic $\langle S, C\rangle$ has the correspondence property iff for every strict homomorphism $h: M \rightarrow N$ between models of $C$ and every filter $F \in F i_{C}(M), F=h^{-1} h(F)$.

Theorem 1. (Blok and Pigozzi [1986], [1992]) For any logic $\langle S, C\rangle$ the following conditions are equivalent:

(i) $\quad C$ is protoalgebraic;

(ii) For all $T, \alpha, \beta: \alpha \equiv \beta(\Omega C(T))$ implies $C(T, \alpha)=C(T, \beta)$;

(iii) The Leibniz operator $\Omega$ is meet-continuous on $T h(C)$;

(iv) $\quad C$ satisfies (R) and (MP) for some $P(p, q)$;

(v) $C$ has the correspondence property;

(vi) $C$ possesses a parameterized system $E(p, q, \underline{r})$ of equivalence sentences;

(vii) The operator $\boldsymbol{\Omega}_{A}$ is monotonic on the lattice $F i_{C}(A)$, for every algebra $A$, i.e., $E \subseteq F$ implies $\boldsymbol{\Omega}_{A} E \subseteq \Omega_{A} F$ for any filters $E, F \in F i_{C}(A)$; 
(viii) The operator $\Omega_{A}$ is meet-continuous on the lattice $F i_{C}(A)$ for every algebra $A$, i.e., $\boldsymbol{\Omega}_{A} \bigcap\left\{F_{i}: i \in I\right\}=\bigcap\left\{\boldsymbol{\Omega}_{A} F_{i}: i \in I\right\}$ for every family $\left(F_{i}\right)_{i \in I}$ of $C$-filters on $A$.

Property (vi) is particularly useful because it implies that for every model $M=\langle A, D\rangle$ for a protoalgebraic logic $C$ :

$$
a \equiv b\left(\boldsymbol{\Omega}_{A} D\right) \Longleftrightarrow \bigwedge_{\underline{e} \in A^{k}} E(a, b, \underline{e}) \subseteq D
$$

where $E(a, b, \underline{e})$ is any $k$-parameterized equivalence for $C$.

There are other characterizations of protoalgebraic logics that give some insight into the behaviour of the monotonic Leibniz operator.

The Deduction Theorem (DT) was independently discovered for classical logic by Herbrand and Tarski. In its paradigmatic formulation it says that:

$$
\beta \in K(X, \alpha) \Longleftrightarrow \alpha \rightarrow \beta \in K(X)
$$

for every set $X$ of sentences and every pair $\alpha, \beta$ of sentences. (Here $K$ is the consequence operation of classical logic and $\rightarrow$ is the material implication.) Since then an array of deduction theorems for various deductive systems has been established. With small exceptions they fall under the general scheme which is discussed below.

Let $\langle S, C\rangle$ be a logic and $p, q$ be fixed distinct variables. Given a set of sentences $P \subseteq S$, we let $\mathrm{MP}_{P}$ denote the detachment rule determined by $P$ :

$\mathrm{MP}_{P}$

$$
\{p\} \cup P / q
$$

The set $P$ is said to have the detachment property for $C$ if $\mathrm{MP}_{P}$ is a rule of $C$, i.e., $q \in C(\{p\} \cup P)$. If $P$ has the detachment property for $C$, then $p$ and $q$ are called main variables while the variables in $\operatorname{Var}(P) \backslash\{p, q\}$ are called parameters of $P$. If $P$ has the detachment property, then so has every superset of $P$.

Let $\boldsymbol{\Phi}$ be a family of sets of sentences $P(p, q, \underline{r})$ involving $k$ parametric variables $\underline{r}=\left\langle r_{0}, r_{1}, \ldots\right\rangle$, where $k \leqslant \omega$.

Definition 3. A logic $\langle S, C\rangle$ is said to admit the parameterized local deduction theorem (PLDT, for short) with respect to $\boldsymbol{\Phi}$ iff for all $T \subseteq S$, and all $\alpha, \beta \in S$ :

PLDT $\quad \beta \in C(T, \alpha)$ iff there exists a set $P(p, q, \underline{r})$ in $\boldsymbol{\Phi}$ and a sequence $\underline{\gamma} \in S^{k}$ of sentences such that $P(\alpha, \beta, \underline{\gamma}) \subseteq C(T)$.

If PLDT holds for all $T, \alpha$ and $\beta$ we say that $\boldsymbol{\Phi}$ determines PLDT for $C$. 
If $C$ admits PLDT with respect to $\boldsymbol{\Phi}$, then each set $P$ in $\boldsymbol{\Phi}$ has the detachment property for $C$. Furthermore, if $C$ is finitary, then $\boldsymbol{\Phi}$ can be required to consist of finite set since for every $P \in \boldsymbol{\Phi}$ a finite set $P_{f} \subseteq P$ can be chosen so that the $\boldsymbol{\Phi}:=\left\{P_{f}: P \in \boldsymbol{\Phi}\right\}$ also determines PLDT for $C$.

Protoalgebraicity and PLDT are equivalent properties:

Theorem 2. (Czelakowski and Dziobiak [1991]) For any logic $C$ the following conditions are equivalent:

(i) $\quad C$ is protoalgebraic;

(ii) $\quad C$ admits PLDT with respect to some family $\boldsymbol{\Phi}$.

PLDT is a very weak form of Deduction Theorem. Specialized instance of PLDT are discussed in Czelakowski's [a].

Protoalgebraic logics can also be characterized in terms of the Suszko operator.

Let $\langle S, C\rangle$ be a logic. For every theory $T \subseteq S$ we define the binary relation $\$ T$ on $S$ by means of the stipulation:

$$
\langle\alpha, \beta\rangle \in \$ T \Longleftrightarrow \bigwedge_{\varphi \in S} \bigwedge_{p \in \operatorname{Var}(\varphi)} C(T, \varphi(p / \alpha))=C(T, \varphi(p / \beta)) .
$$

The definition of $\$ T$ is strictly relativised to the logic $C$ and, unlike the definition of the Leibniz congruence, it does not have the absolute character - $\$ T$ may vary on passing from $C$ to a weaker logic. $\$ T$ can be shown to be a congruence on $S$ compatible with $T$. Therefore $\$ T \subseteq \Omega T$, for all $T \in T h(C)$ and this inclusion may be proper unless $C$ is protoalgebraic (see Thm. 3 below). The congruence $\$ T$ is called the Suszko congruence corresponding to the theory $T$. $\$ T$ is the largest congruence $\boldsymbol{\Phi}$ on $S$ with the property that $\alpha \equiv \beta(\boldsymbol{\Phi})$ implies that $C(T, \alpha)=C(T, \beta)$, for all $\alpha, \beta$. The operator $\$$ which to each theory $T \in T h(C)$ assigns the congruence $\$ T$ is called the Suszko operator. The condition on the right-side of $(* *)$ was used by Suszko to define the identity connective in his sentential logic with identity. It follows from the definition of $\$$ that, for any $\operatorname{logic} C$, not necessarily protoalgebraic, the operator $\$$ is monotonic on $T h(C)$, i.e., $\$ T_{1} \subseteq \$ T_{2}$ whenever $T_{1} \subseteq T_{2}$.

The domain of the operator $\$$ is extended onto the power set of an arbitrary algebra $A$ similar to $S$. For every set $D \subset A$, the relation $\$_{A} D$ is defined as follows:

$$
\bigwedge_{\varphi\left(p, r_{1}, \ldots, r_{k}\right) \in S} \bigwedge_{\left\langle e_{1}, \ldots, e_{k}\right\rangle \in A^{k}} C_{A}\left(D, \varphi\left(a, e_{1}, \ldots, e_{k}\right)\right)=C_{A}\left(D, \varphi\left(b, e_{1}, \ldots, e_{k}\right)\right) .
$$

Here $C_{A}(X)$ is the $C$-filter on $A$ generated by the set $X \subseteq A$. 
$\$_{A} D$ is a congruence on $A$ compatible with the $C$-filter $C_{A}(D)$, for all $D \subseteq A$. Furthermore, if $E \subseteq F$, then $\$ E \subseteq \$ F$, for all $E, F \subseteq A$, and $a \equiv b$ $\left(\$_{A} D\right)$ implies $C_{A}(D, a)=C_{A}(D, b)$, for all $a, b \in A$.

Theorem 3. (Czelakowski [1992]) Let $\langle S, C\rangle$ be an arbitrary logic and let $A$ be an algebra similar to $S$. Suppose that an operator $\boldsymbol{O}$, defined on $F i_{C}(A)$, has the following property:

$$
a \equiv b(\boldsymbol{O} D) \text { implies that } C_{A}(D, a)=C_{A}(D, b),
$$

for all $D \in F i_{C}(A)$ and $a, b \in A$. Then $\boldsymbol{O} D \subseteq \$ D$, for every $D \in F i_{C}(A)$.

The above theorem thus states that the Suszko operator is the largest of all operators $\boldsymbol{O}$ defined on $F i_{C}(A)$ for which $(\star)$ holds.

The Suszko and Leibniz operators coincide for $C$ protoalgebraic. This property, in fact, characterizes protoalgebraic logics:

Theorem 4. (Czelakowski [1992]) For a logic $\langle S, C\rangle$ the following conditions are equivalent:

(i) $C$ is protoalgebraic;

(ii) $\$ T=\Omega T$, for every theory $T \in T h(C)$;

(iii) $\$_{A} D=\Omega_{A} D$, for every algebra $A$ similar to $S$ and $D \in F i_{C}(A)$.

\section{Equivalential logics}

According to Theorem 1, each protoalgebraic logic $C$ is characterized by the existence of a possibly infinite system of parameterized equivalence sentences $E(p, q, \underline{r})$. Equivalential logics are defined (Prucnal and Wroński [1974]) in terms of the existence of a parameter-free system of equivalence sentences. This class encompasses almost all non-trivial deductive systems which have been studied in the literature.

Definition 4. (Prucnal and Wroński [1974]) A logic $\langle S, C\rangle$ is equivalential (finitely equivalential, respectively) iff $C$ has a parameter-free system $E(p, q)$ (a finite parameter-free system $E(p, q)$ ) of equivalence sentences, i.e., the following conditions are met for some set (finite set) $E(p, q)$ :

$$
\begin{aligned}
& E(p, p) \subseteq C(\emptyset) ; \\
& q \in C(E(p, q) \cup\{p\}) ;
\end{aligned}
$$


$\left(\mathrm{RP}_{\text {sim }}\right) \quad$ for each $n$-ary connectives $f$ of $S(n \geqslant 0)$ and two $n$-tuples of disjoint variables $p_{1}, \ldots, p_{n}, q_{1}, \ldots, q_{n}$

$$
E\left(f\left(p_{1}, \ldots, p_{n}\right), f\left(q_{1}, \ldots, q_{n}\right)\right) \subseteq C\left(E\left(p_{1}, q_{1}\right) \cup \ldots \cup E\left(p_{n}, q_{n}\right)\right) .
$$

The set $E(p, q)$ is then called an equivalence system for $S$ (cf. Theorem 1. (vi)).

The above three conditions are called reflexivity, Modus Ponens, and simple replacement. They are known to imply the conditions of symmetry and transitivity (see Wójcicki [1988], p. 223).

Definition 4 and Theorem 1. (vi) imply that every equivalential logic is protoalgebraic. Equivalential logics can be characterized in terms of the Leibniz operator. (In fact, each of the conditions given below might be used as a definition of the class of equivalential logics.)

Theorem 5. (Czelakowski [1992], Herrmann [1993], Jansana) For every logic $\langle S, C\rangle$ the following conditions are equivalent:

(i) $\quad C$ is equivalential;

(ii) The operator $\boldsymbol{\Omega}$ is monotonic and commutes with inverse substitutions on $T h(C)$, i.e., $e^{-1} \boldsymbol{\Omega} T \subseteq \boldsymbol{\Omega} e^{-1} T$ for any substitution $e$ in $S$ and any $T \in T h(C)$;

(iii) $\boldsymbol{\Omega}$ is monotonic and $e \boldsymbol{\Omega} T \subseteq \boldsymbol{\Omega} C(e T)$ for all substitutions $e$ and all $T \in T h(C)$;

(iv) $\Omega$ is $\sigma$-continuous ${ }^{1}$ on $F i_{C}(A)$, for every algebra $A$ similar to $S$.

The finitely equivalential logics are characterized in terms of the Leibniz operator:

Theorem 6. (Blok and Pigozzi [1992], Czelakowski [1992], Herrmann [1993]) For any logic $\langle S, C\rangle$ the following conditions are equivalent:

(i) $\quad C$ is finitely equivalential;

(ii) $\boldsymbol{\Omega}$ is continuous on $T h(C)$;

(iii) $\boldsymbol{\Omega}$ is continuous on $F i_{C}(A)$, for every algebra $A$ similar to $S$.

${ }^{1}$ An operator $\boldsymbol{O}$ is $\sigma$-continuous on $F i_{C}(A)$ iff

$$
\boldsymbol{O} \bigcup\left\{D_{i}: i \in I\right\}=\bigcup\left\{\boldsymbol{O} D_{i}: i \in I\right\}
$$

for every $\sigma$-directed family $\left(D_{i}\right)_{i \in I}$ of $C$-filters of $A$.

The fact that $\left(D_{i}\right)_{i \in I}$ is $\sigma$-directed family means that for every countable subset $K \subseteq I$, there exists a $k \in I$ such that $\bigcup\left\{T_{i}: i \in K\right\} \subseteq T_{k}$. 
We have briefly discussed two classes of logics forming the lowest levels in the hierarchy of deductive systems: protoalgebraic logics and equivalential logics. The next three levels are occupied by the classes of algebraizable logics, introduced by Blok and Pigozzi [1989], regularly algebraizable (alias 1-algebraizable) logics, introduced by Czelakowski [1981], and the class of protoalgebraic Fregean logics, studied by Pigozzi, Czelakowski, Font, Jansana and Herrmann. They form a descending chain of classes of deductive systems. We thus have the following inclusions:

\section{Protoalgebraic logics $\supset$ Equivalential logics $\supset$ Algebraizable logics $\supset$ Regularly algebraizable logics $\supset$ Protoalgebraic Fregean logics.}

We cannot give here the definition of an algebraizable logic. We only mention here that a class $\mathcal{K}$ of algebras similar to $S$ is called an algebraic semantics for a logic $\langle S, C\rangle$ iff $C$ can be interpreted in a natural way, in the „equational" consequence operation $\mathcal{K}^{\text {eq }}=$ determined by $\mathcal{K}$ on the equational language corresponding to $\mathcal{K}$. If conversely, $\mathcal{K}^{\mathrm{eq}} \models$ can be reconstructed on the basis of $C$, the class $\mathcal{K}$ is called an equivalent algebraic semantics for the logic $C$. The logic $C$ is algebraizable in the strict sense iff it possesses an equivalent algebraic semantics. (The notion of algebraizability is being understood here more widely than in Blok and Pigozzi [1989] since the class of algebraizable logics may contain infinite logics. Their original definition is restricted to finitary deductive systems.) The above, apparently complicated definitions, render the essence of the procedure of assigning the class of Lindenbaum-Tarski algebras to a given logic.

Theorem 7. (Blok and Pigozzi [1989], Czelakowski [1992], Herrmann [1993]) Let $\langle S, C\rangle$ he a sentential logic. The following conditions are equivalent:

(i) $\quad C$ is algebraizable;

(ii) The operator $\Omega$ is injective, monotonic and commutes with inverse substitutions on $T h(C)$;

(iii) $C$ is equivalential and $\boldsymbol{\Omega}$ is injective on $T h(C)$.

(In the above theorem, $C$ is not assumed to be finitary, and consequently algebraizability is understood here wider than in Blok and Pigozzi [1989].)

A logic $\langle S, C\rangle$ is regularly algebraizable iff it is equivalential (and thus it has an equivalence system $E(p, q))$ and $p, q / E(p, q)$ is a set of rules of $C$ for some (equivalently, for any) equivalence system $E(p, q)$. In other words, regularly algebraizable logics are equivalential logics in which the members 
of an arbitrary theory $T$ are all identified under the relation $\boldsymbol{\Omega} T$. Regularly algebraizable logics are thus characterized as equivalential $\operatorname{logics} C$ for which the reduced (alias factorial) models from the class $\operatorname{Mod}^{*}(C)$ have the one-element set of designated elements. (Each such reduced model is customarily denoted by $(A, 1)$; this justifies the use of the term '1-algebraizable logic'. Every regularly algebraizable logics is algebraizable, but not vice versa (see Blok and Pigozzi [1989]). Regularly algebraizable logics encompass implicative calculi in the sense of Rasiowa [1974]. Regularly algebraizable logics are characterized in terms of the Leibniz operator as equivalential logics in which $\alpha \equiv \beta(\boldsymbol{\Omega} C(\alpha, \beta))$ for any sentences $\alpha, \beta$.

The origin of non-Fregean logics stems from the critique and abolition of Frege axiom by Suszko [1968], [1975]. Acceptance of this axiom leads to distinguishing the class of Fregean logics. Formally, a protoalgebraic logic $C$ is Fregean iff $C$ is not almost inconsistent (i.e., $C$ does not satisfy: $C(\emptyset)=\emptyset$ $\& C(X)=S$ whenever $X$ is non-empty) and the Leibniz operator $\boldsymbol{\Omega}$ satisfies the condition:

$$
\alpha \equiv \beta(\Omega C(T)) \Longleftrightarrow C(T, \alpha)=C(T, \beta),
$$

for all $T, \alpha$ and $\beta$.

Classical and intuitionistic logics are Fregean since $(\star \star)$ reduces for these logics to the well-known Tarski's condition:

$$
\alpha \leftrightarrow \beta \in C(T) \Longleftrightarrow C(T, \alpha)=C(T, \beta),
$$

for all $T, \alpha$ and $\beta$. Every Fregean protoalgebraic logic $C$ is regularly algebraizable but not vice versa. E.g. the three-valued Łukasiewicz logic is regularly algebraizable; it is not Fregean. Quantum logics, i.e., the consequence operations $C$ determined by classes of orthomodular lattices with the unit element designated, are known to be regularly algebraizable and non-Fregean unless the logic $C$ coincides with the classical consequence (Czelakowski and Pigozzi - an unpublished result).

\section{Final remarks}

The class of protoalgebraic logic is too restrictive, at least from the viewpoint of metalogic. E.g. the conjunctive-disjunctive fragment of classical logic falls outside this class. It is not difficult to show that the protoalgebraic logics determined by two-element truth-tables are extensions of either the implicational or the equivalential fragment of classical logic (they may involve more connectives than $\rightarrow$ or $\leftrightarrow$ ). This gives rise to the problem of working out 
a more general conceptual apparatus which would make all logics, not only protoalgebraic ones, amenable to its methods. An instrument of this kind is available - the key role is played here by the Suszko operator. We know that for protoalgebraic logics, the Suszko and Leibniz operators coincide. The list of plausible properties of the Suszko operator, parallel to those studied for the Leibniz operator, may serve as a basis for distinguishing a hierarchy of all logics which, in turn, parallels the hierarchy of protoalgebraic logics discussed above and, at the same time, is an extension of the above protoalgebraic logics hierarchy. A significant role in clarifying the basic notions of this extended hierarchy is played by the notion of a matrix reduced in the sense of Suszko, shortly, a Suszko-reduced matrix. A matrix $M=\langle A, D\rangle$ is Suszko-reduced iff the congruence $\$_{A} D$ is the identity relation on the algebra $A$. Since the Suszko operator is always monotonic, the class $\operatorname{Mod}^{\$}(C)$ of Suszko-reduced matrices for any logic $C$ is closed under the formation of subdirect products (Czelakowski [1992]). This fact makes it possible to use widely the techniques of algebraic provenience in metalogical investigations of whatever logics.

\section{References}

Blok, W. J. and Pigozzi, D. [1986] „Protoalgebraic logics”, Studia Logica 45, 337-369.

Blok, W. J. and Pigozzi, D. [1989] Algebraizable Logics, Memoirs of the Amer. Math. Soc. No. 396, Amer. Math. Soc., Providence.

Blok, W. J. and Pigozzi, D. [1992] „Algebraic semantics for universal Horn logic without quality", [in:] Universal Algebra and Quasigroups, (eds. A. Romanowska and J. D. H. Smith), Heldermann Verlag, Berlin.

Czelakowski, J. [1981] „Equivalential logics: (I), (II)”, Studia Logica 40, 227-236, $335-372$.

Czelakowski, J. [1992] Consequence Operations. Foundational Studies, Polish Academy of Sciences, Warsaw.

Czelakowski, J. [a] „Protoalgebraic logics”, to appear.

Czelakowski, J. and Dziobiak, W. [1991] „A deduction theorem scheme for deductive systems of propositional logics", Studia Logica 50, Special Issue on Algebraic Logic (eds. W. J. Blok and Don Pigozzi), 385-390.

Herrmann, B. [1993] Equivalential Logics and Definability of Truth, Ph. Dissertation, Freie Universität Berlin. 
Łoś, J. [1949] On Logical Matrices (Polish), Travaux de la Sociéte les Sciences et des Lettres de Wrocław, Seria B, Nr 19, Wrocław.

Pigozzi, D. [1991] „Fregean algebraic logic”, [in:] Algebraic Logic (Proc. Conf. Budapest, 8-14 August 1988), (eds. H. Andréka, J. Monk and I. Németi), Colloq. Math. Soc. J. Bolyai, Vol. 54, North-Holland, Amsterdam, 473-502.

Prucnal, T. and Wroński, A. [1974] „An algebraic characterization of the notion of structural completeness", Bulletin of the Section of Logic 3, Polish Academy of Sciences, 30-33.

Rasiowa H. [1974] An Algebraic Approach to Non-Classical Logics, PWN and North-Holland, Warsaw - Amsterdam.

Suszko, R. [1968] „Ontology in the Tractatus of L. Wittgenstein”, Notre Dame Journal of Formal Logic 9, 7-33.

Suszko, R. [1975] „Abolition of the Fregean axiom”, [in:] Logic Colloquium (Boston, Mass, 1972-1973), (ed. R. Parikh), Lecture Notes in Mathematics 453, Springer Verlag, Berlin, 169-236.

Wójcicki, R. [1973] „Matrix approach in sentential calculi”, Studia Logica 32, 7-37.

Wójcicki, R. [1988] Theory of Logical Calculi. Basic Theory of Consequence Operations, Kluwer, Dordrecht.

\author{
JANusz CzELAKowski \\ Institute of Mathematics \\ University of Opole \\ ul. Oleska 48 \\ 45-052 Opole, POLAnd \\ e-mail: janusz.czelakowski@uni.opole.pl
}

